\title{
Die Endoprothetik des oberen Sprunggelenkes
}

\author{
Jürgen Rudigier, Felix Menzinger
}

\section{Zusammenfassung}

In der Versorgung posttraumatischer Arthrosen des oberen Sprunggelenkes haben die seit Beginn der 90er-Jahre verfügbaren zementfreien Sprunggelenksendoprothesen die Indikation zur operativen Versteifung (Arthrodese) zunehmend infrage gestellt und im eigenen Krankengut auf wenige Sonderfälle zurückgedrängt. Die eigenen Erfahrungen seit mehr als 13 Jahren bei inzwischen über 100 Patienten zeigen, dass mehr als 75\% eine nahezu normale Gehfähigkeit wiedererlangen und die übrigen wesentliche
Verbesserungen erfahren, so dass von mehr als $90 \%$ guten und sehr guten $\mathrm{Er}$ gebnisse gesprochen werden kann, wie dies auch andere Autoren mit ähnlich aussagekräftigen Patientenkollektiven belegen [6]. Bei komplexen Arthrosen, Fehlstellungen und defekter Sprunggelenksgabel sind allerdings nicht alle Prothesenmodelle gleich gut geeignet und besondere operative Erfahrungen notwendig. Langzeitbeobachtungen und Verbesserungen in Material und Design lassen erwarten, dass mit Überlebenszeiten der Endoprothesen von 20 und mehr Jahren zu rechnen ist.

\section{Einleitung}

Die ersten Sprunggelenksendoprothesen wurden bereits Anfang der 70er Jahre implantiert $[6,7,9]$.

Dass sich die Sprunggelenksendoprothetik zunächst nicht als Standardverfahren etablieren konnte, ist vor allem darin begründet, dass die Endoprothesen bis zu Beginn der 70er-Jahre überwiegend mit Hilfe von Knochenzement verankert wurden, wodurch die Tragfähigkeit des Knochenlagers erheblich geschwächt wurde, und dass das Prothesendesign nicht ausreichend die Scherkräfte an der Implantat-Knochengrenze berücksichtigte. Wegen der hierdurch bedingten Fehlschläge (Lockerung, Einsinken der Prothesen, Knochennekrosen und Infektionen) wurde daher, vor allem in Deutschland, die primäre Arthrodese des oberen Sprunggelenkes als Methode der Wahl bevorzugt $[1,3,4]$.

OP-JOURNAL 2003; 19: 230-235

(c) Georg Thieme Verlag Stuttgart . New York
Nachdem seit etwa 1990 zunehmend zementfreie Implantationen erfolgten (aktuell werden alle im Handel befindlichen Endoprothesen zementfrei implantiert), hat sich diese Situation grundlegend geändert. Lockerungen und Wanderungen sind wesentlich seltener geworden, Knochennekrosen oder gar das Ausbrechen der Prothese aus dem Knochenlager kommen kaum noch vor $[6,9]$.

Daher ist die Endoprothetik des oberen Sprunggelenkes inzwischen nicht nur eine empfehlenswerte Alternative zur Arthrodese des oberen Sprunggelenkes geworden, sondern sie stellt vielfach das bessere Verfahren dar.

Dies betrifft neben der besseren und schmerzfreien Gehfähigkeit vor allem das Vermeiden nachfolgender Arthrosen in den Nachbargelenken.

\section{Modelle}

Zu Verfügung stehen 2 Arten von Modellen (Abb.1):

1. Endoprothesen mit 3 gegeneinander beweglichen Komponenten (Menis- kusendoprothesen), bei denen sich zwischen den fest am Knochen verankerten Tibia- und Talusteilen ein Polyethylengleitkern (Meniskus) bewegen kann (Abb.1a). Dessen Beweglichkeit neutralisiert bei diesen Prothesen teilweise die beim Gehvorgang auftretenden Scherkräfte an der Knochenzementgrenze und es können eventuelle Rotationsfehler ausgeglichen werden. Ein Nachteil bei diesen Endoprothesen ist allerdings, dass unbedingt intakte Gabelverhältnisse vorliegen müssen und dass das Einstellen einer guten Balance zwischen den 3 Komponenten schwierig sein kann.

Die erste derartige Prothese ist die sogenannte

Büschel-Papas-Endoprothese (USA),

die bereits zementfrei seit 1981 implantiert wird (Publikation erst 1989) [2\}.

Eine skandinavische Meniskusendoprothese, die

\section{STAR-Endoprothese,}

war bereits als zementiertes Modell zunächst in einer 2-Komponenten-Ausführung Ende der 70er-Jahre auf dem Markt, damals mit allen Problemen der zementierten Endoprothese. Die Ergebnisse besserten sich durch Änderung in eine 3-Komponenten-Prothese (1984) und Einführen der zementfreien Implantation seit 1990 [6].

Einige weitere auf dem Markt befindliche Prothesen sind vom Prinzip dieser beiden Prothesentypen abgeleitet (z.B. Salto, 1995 Frankreich, Hintegra, 1999 Schweiz) [10].

2. 2-Komponenten-Prothesen, die vom Grundkonzept her auf einen beweglichen Meniskus zwischen Tibia- und Taluskomponente verzichten (Abb.1 b). 


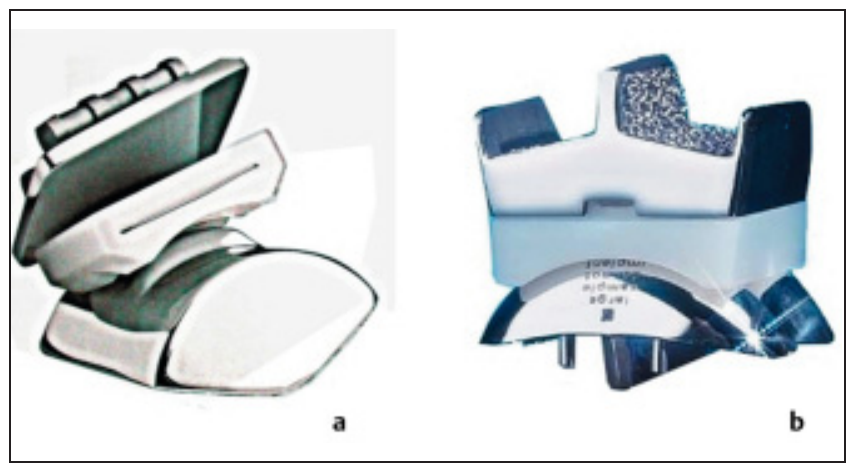

Abb.1 Endoprothesenmodelle, (a) Beispiel für 3-Komponenten- oder Meniskus-Endoprothesen mit einem beidseits beweglichen Polyethylenmittelteil (STAR-Endoprothese), (b) Beispiel für eine 2-KomponentenEndoprothese mit am Tibiateil fixierten Polyetylenmittelteil (ESKA-Endoprothese)

\section{Hierzu zählt die}

\section{Agilityprothese}

(1985 USA), bei der die Sprunggelenksgabel weitgehend entfernt wird, um über eine Fusion zwischen Talus und Tibia eine große Auflagefläche für die Tibiakomponente zu erreichen [10].

Neu bezüglich des Verankerungskonzeptes war die

\section{ESKA-Endoprothese}

(1990 Deutschland), bei der die Entstehung von Scherkräften durch eine firstartige Gestaltung der Oberflächen im Tibiaund Talusteil verhindert werden [9]. Diese Endoprothese, die bei gleicher Grundkonzeption 1993 und 1998 strukturelle Verbesserungen erfuhr, implantieren wir seit 1990 mit bestem Erfolg bei unserem vorwiegend posttraumatischen Krankengut (Abb.1b). Sie weist gegenüber Meniskusprothesen den Vorteil auf, unabhängig von intakten Verhältnissen der Sprunggelenksgabel zu sein, wodurch die Indikation wesentlich weiter gestellt werden kann. Gegenüber der Agilityendoprothese ist der Knochensubstanzverlust geringer und die Koppelung zwischen Tibia- und Talusteil stabiler.

\section{Implantationshäufigkeit}

In der eigenen Klinik hat sich die Zahl der implantierten ESKA-Endoprothesen von ursprünglich 3-5 pro Jahr auf 20 bis 30 Patienten pro Jahr gesteigert (Abb.2).

Fasst man alle gängigen Sprunggelenksprothesentypen zusammen, dürfte die
Zahl der Implantationen zwischen 500 und 700 pro Jahr in Deutschland betragen (genaue statistische Zahlen fehlen, die Angaben beziehen sich auf Auskünfte der Hersteller). Weltweit schätzen wir aufgrund der zur Verfügung stehenden Literatur und Kongressberichte die Zahl der jährlich implantierten Sprunggelenksprothesen auf 3000-5000 pro Jahr.

\section{Indikationsstellung}

Die Sprunggelenksendoprothetik konkurriert mit dem Verfahren der Arthrodese. Vor- und Nachteile der Prothese gegenüber der Arthrodese sind daher abzuwägen [5,8]. Die Endoprothetik erlaubt in den meisten Fällen ein besseres schmerzfreies Gehen und die Vermeidung von Sekundärarthrosen, sofern eine gute Beweglichkeit nach der Prothesenimplanta-

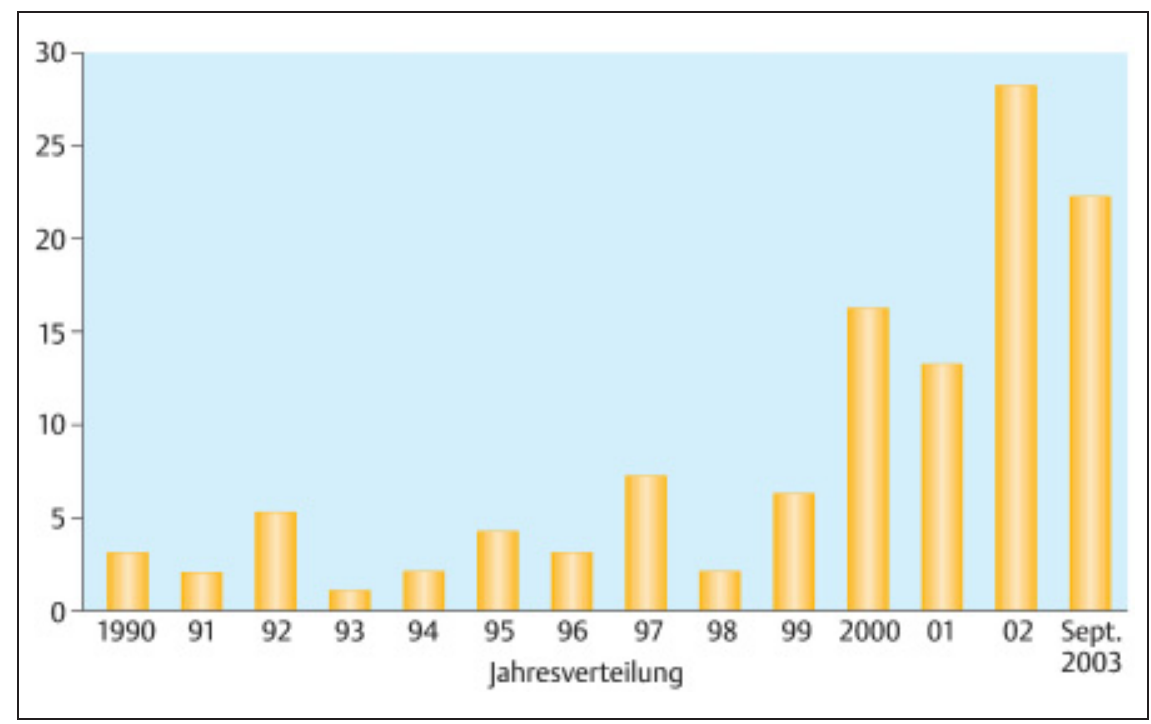

Abb.2 Zunahme der Implantationshäufigkeit im eigenen Krankengut seit 1990. tion erreicht wird. Der Vorteil der verbesserten Beweglichkeit kann aber gelegentlich, zumindest für einen gewissen Zeitraum, mit zusätzlichen Schmerzen im Kapselbandbereich erkauft werden, bis sich die Situation an die verbesserte Beweglichkeit angepasst hat. Diese Problematik besteht bei der Arthrodese nicht.

Die Hauptindikation zur Endoprothesenimplantation ist wie bei der Arthrodese die schmerzhafte Arthrose des oberen Sprunggelenkes nach Ausschöpfen aller gelenkerhaltender Maßnahmen.

Problematisch und damit Kontraindikationen sind:

zusätzliche neurologische oder psychiatrische Erkrankungen,

- Situationen mit völlig eingesteiftem Sprunggelenk und bereits eingetretenen Sekundärarthrosen in den Nachbargelenken (kein Vorteil gegenüber Arthrodese!),

- fehlende Fußpulse (arterielle Verschlusskrankheit),

- extreme Osteoporose (Arthrodese ebenfalls schwierig),

- hohes Lebensalter mit geringer Gehfähigkeit und Polymorbidität (häufig auch eine Kontraindikation gegen eine Arthrodese).

- Defekte im Außen- oder Innenknöchelbereich sind für Meniskusendoprothesen eine Kontraindikation ebenso wie größere Achsenfehler. Beides gilt nicht für die von uns seit 1990 implantierte ESKA-Endoprothese!

Bei Knochennekrosen oder Knochendefekten kann ein präoperatives Kernspin- 


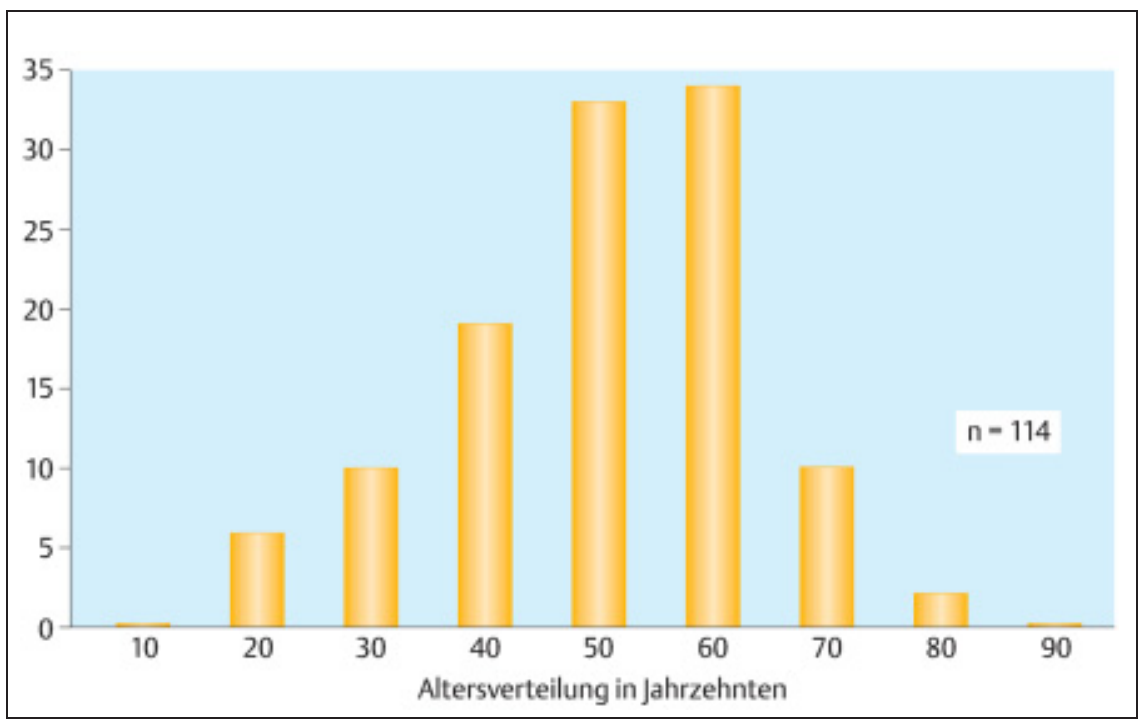

Abb. 3 Häufigkeit der Implantationen nach Lebensalter.

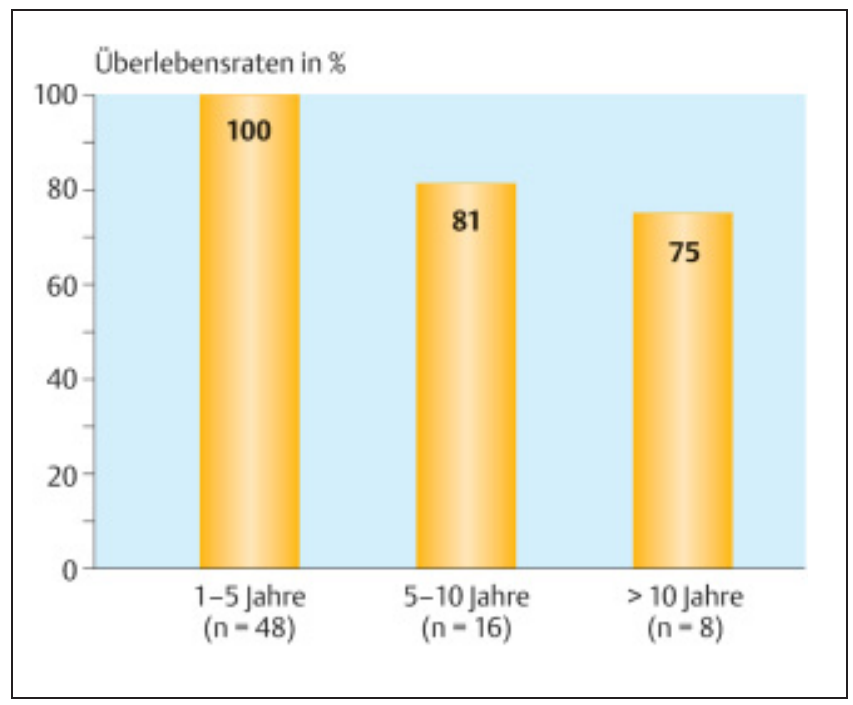

Abb. 4 Intakte Endoprothesen nach Implantationsdauer (eigenes Krankengut in \%). tomogramm klären, ob ausreichend tragende Knochensubstanz in Talus oder Tibia vorhanden ist. Eventuelle Defekte und Zysten können intraoperativ mit anfallendem Knochenmaterial ausgefüllt werden.

\section{Operatives Vorgehen}

Die Meniskusendoprothesen werden mithilfe verschiedener Zielgeräte und Schablonen über einen ventralen Zugang implantiert, da die Sprunggelenksgabel intakt bleiben muss.

Das von uns favorisierte 2-Komponenten-Modell wird hingegen wegen seiner firstartigen Implantatoberfläche von einem ventro-lateralen Zugang aus eingebracht (9). erforderlich. Es reicht aus, wenn nach der Außenknöchelosteosynthese mit einem Drittelrohrplättchen das am ventralen Weichteillappen erhalten gebliebene Kapselgewebe transossär am Außenknöchel refixiert wird.

Die Nachbehandlung besteht in einer 2wöchigen Ruhigstellung mit einem geschalten Unterschenkelgips in $90^{\circ}$ Dorsalextension. Ab dem 6 . Tag wird $1 \times$ täglich die Gipsschiene entfernt und aktiv und passiv beübt. Nach Entfernen der Hautfäden erfolgt die Entlassung des Patienten mit einem Gehgips, der voll belastet werden darf. Zu Beginn der 7. postoperativen Woche wird der Gehgips entfernt und die Belastung auf das halbe Körpergewicht zurückgenommen. Der Patient steigert danach wöchentlich unter Verwendung von 2 Unterarmgehstützen die Belastung um $10-20 \mathrm{~kg}$. Meist ist nach 12 Wochen die volle Belastung erreicht. Ab diesem Zeitpunkt gehen ca. $2 / 3$ der Patienten ohne Unterarmgehstützen. Einzelne Patienten benützen noch 1-2 Monate länger einen Gehstock.

Probleme durch die zugangsbedingte Osteotomie des Außenknöchels waren nicht zu beobachten. Sie heilte meist innerhalb von 6-8 Wochen, gelegentlich nach einigen Monaten (ohne Konsequenzen für die Nachbehandlung).

\section{Ergebnisse}

Insgesamt überblicken wir eine Zahl von 116 Patienten (13 dieser Patienten wurden unter unserer Mitwirkung in benachbarten Kliniken operiert, 105 Patienten werden in der eigenen Klinik nachbetreut).

Am häufigsten sind Patienten zwischen 50 und 70 Jahren (jüngster Patient 22 Jahre, ältester 82 Jahre) (Abb.3).

$63 \%$ hatten eine unfallbedingte Arthrose (z.B. Abb. 5 u. 6), 25\% eine Arthrose unbekannter Ursache und $12 \%$ eine chronische Polyarthritis.

Bei 3 Patienten wurden gescheiterte Arthrodesen mit Endoprothesen versorgt. Bei 6 Patienten lagen präoperativ erhebliche Achsenfehler im OSG vor.

Bei 9 Patienten kennen wir den Verlauf über mehr als 10 Jahre, bei insgesamt 27 Patienten mehr als 5 Jahre. 26 Patienten mit einer Implantationsdauer unter 12 Monaten bleiben bei der Darstellung der Ergebnisse unberücksichtigt.
Eine Rekonstruktion der Syndesmose is wegen der guten Führung der Prothesenkomponenten in sagittaler Richtung nicht 

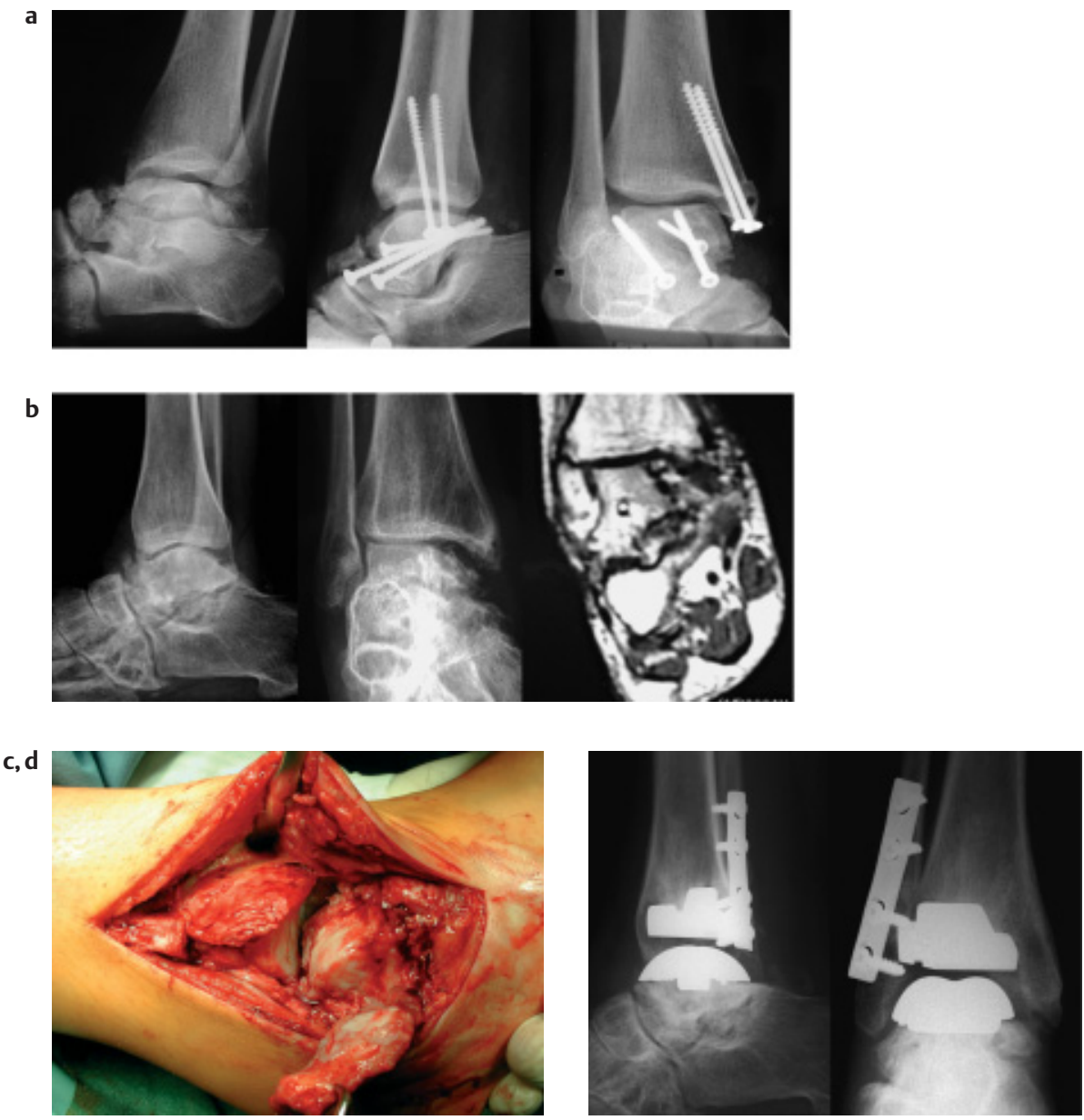

Abb.5 22 Jahre alte Patientin nach Talustrümmerfraktur, (a) Unfall- und Versorgungsbilder, (b) präoperative Talusteilnekrose (Dauerschmerz, Instabilität) c OP-Situs mit weggeklapptem Außenknöchel und guter Sicht auf die Gelenkflächen, (d) 16 Monate nach Operation (durch Endoprothese wieder voll arbeitsfähig als Arzthelferin, schmerzfrei, unbegrenzte Gehstrecke, Beweglichkeit $\left.5 / 0 / 40^{\circ}\right)$.

Score: Der aus Gründen der Vergleichbarkeit mit anderen Autoren verwendete Score nach Kofoed, ergab im Gesamtkrankengut einen Wert von durchschnittlich 91 Punkten postoperativ, gegenüber einer Ausgangssituation von 35 Punkten. Die Werte nach 1 Jahr Implantationsdauer und mehr als 5 Jahren Implantationsdauer unterscheiden sich interessanterweise nur um etwa 1 Punkt (Scorekriterien: Schmerz, Funktion, Beweglichkeit. Bewertung: sehr gut 85-100 Punkte, gut 75-84 Punkte, befriedigend 70-74 Punkte, unzureichend $<70$ Punkte).

Besser als jeder Score ist zur objektiven Beurteilung der erzielten Ergebnisse die Betrachtung folgender Kriterien geeignet: Schmerzsituation, Beweglichkeit des Gelenkes, Gehstrecke und Überlebensdauer des Kunstgelenkes (Tab.1, Abb.4).
Schmerz: 1 Jahr nach der Implantation waren Dauerschmerzen bei allen Patienten, bei denen die Prothese komplikationslos einheilte, beseitigt. Zeitweise Schmerzen nach längerer Belastung oder unklare Schmerzen wechselnder Intensität mit schmerzfreien Intervallen gaben noch $26 \%$ der Patienten (von ursprünglich $100 \%$ ) an, über Anlaufschmerzen (die ersten 3 bis 10 Schritte sind nach längerer Ruhe schmerzhaft, danach wird schmerzfreies Gehen möglich) klagten noch $6 \%$ der Patienten.

Gehstrecke: Die Gehstrecke betrug präoperativ bei $80 \%$ der Patienten weniger als $1 \mathrm{~km}$, postoperativ nur noch bei $5 \%$, die in aller Regel zusätzliche Probleme in anderen Gelenken aufwiesen.

29\% der Patienten konnten postoperativ zwischen 1 und $5 \mathrm{~km}$, meist um die $3 \mathrm{~km}$ herum, schmerzfrei gehen.
66\% der Patienten erreichten postoperativ schmerzfreie Gehstrecken von mehr als $5 \mathrm{~km}$ (Abb.5 u.6).

Beweglichkeit: Präoperativ betrug das Ausmaß der Beweglichkeit des oberen Sprunggelenkes bei $71 \%$ der Patienten weniger als $20^{\circ} .17 \%$ besaßen noch eine akzeptable Beweglichkeit von $20^{\circ}-39^{\circ}$ und nur $12 \%$ hatten eine annähernd normale Beweglichkeit von mehr als $40^{\circ}$.

Postoperativ verbesserte sich durchschnittlich das Bewegungsausmaß um etwa $20^{\circ}$. In der Gruppe der Minimalbeweglichkeiten verblieben nur $10 \%$ der Patienten, 57\% der Patienten erreichten Beweglichkeiten von $20^{\circ}-40^{\circ}$ und $33 \%$ der Patienten Beweglichkeiten von mehr als $40^{\circ}$.

Überlebensdauer (Abb.4): Bezogen auf 105 Patienten wurde 4-mal ein Prothesenausbau mit Umwandlung in eine Arthrodese des oberen Sprunggelenkes durchgeführt, und 4-mal erfolgten Endoprothesenwechsel.

Über 10 Jahre Implantationsdauer: In der Gruppe der 11 Implantationen zwischen 1990 und 1993 ist ein Patient nach 4 Jahren bei intakter Endoprothese gestorben, bei zwei Patienten wurde die Endoprothese in eine Arthrodese umgewandelt wegen nicht anders beherrschbarer Infekte (beide hatten bereits im Bereich anderer Endoprothesen eine Infektanamnese), bei einem Patienten erfolgte eine Umwandlung in eine Arthrodese wegen weitergehender rheumatischer Destruktion im Talusbereich nach 11 Jahren und bei unserer ersten Endoprothese wurde wegen Materialermüdung bei übergewichtiger Patientin nach 9 Jahren ein Wechsel durchgeführt.

Schließt man die beiden Protheseninfekte und den verstorbenen Patienten aus, so sind von 8 implantierten Endoprothesen nach über 10 Jahren noch 6 mit guter Funktion intakt (75\%).

5-10 Jahre Implantationsdauer: In der Gruppe der Patienten zwischen 1994 und 1998 sind 16 Patienten in unserer Nachkontrolle. Ein Prothesenwechsel erfolgte nach 7 Jahren (Spätfolgen eines Implantationsfehlers!), ein Wechsel nach 4 Jahren (Lockerung bei großer mit Spongiosa aufgefüllter Knochenzyste im Tibiabereich) sowie ein Wechsel nach 7 Jahren wegen eines hämatogen entstandenen Gelenkempyems. 


\begin{tabular}{|c|c|c|}
\hline Kofoed-Ankle-Score: & präoperativ & postoperativ \\
\hline $1 \mathrm{Jahr}$ & 34,75 & 91,25 \\
\hline $1-5$ Jahre & 43,21 & 90,03 \\
\hline$>5$ Jahre & 32,25 & 89,88 \\
\hline total $(n=72)$ & 36,51 & 90,24 \\
\hline Schmerzen $(n=72)$ & präoperativ & postoperativ \\
\hline dauernd & 58 & 0 \\
\hline bei Belastung & 72 & 19 \\
\hline Anlaufschmerzen & 72 & 4 \\
\hline keine & 0 & 49 \\
\hline Gehstrecken $(n=72)$ & präoperativ & postoperativ \\
\hline$<1000 \mathrm{~m}$ & 57 & 4 \\
\hline $1000-5000 m$ & 13 & 21 \\
\hline$>5000 \mathrm{~m}$ & 2 & 47 \\
\hline Bewegungsausma $ß(n=72)$ & präoperativ & postoperativ \\
\hline $0-20^{\circ}$ & 51 & 7 \\
\hline $20-39^{\circ}$ & 12 & 41 \\
\hline $40-60^{\circ}$ & 9 & 24 \\
\hline
\end{tabular}
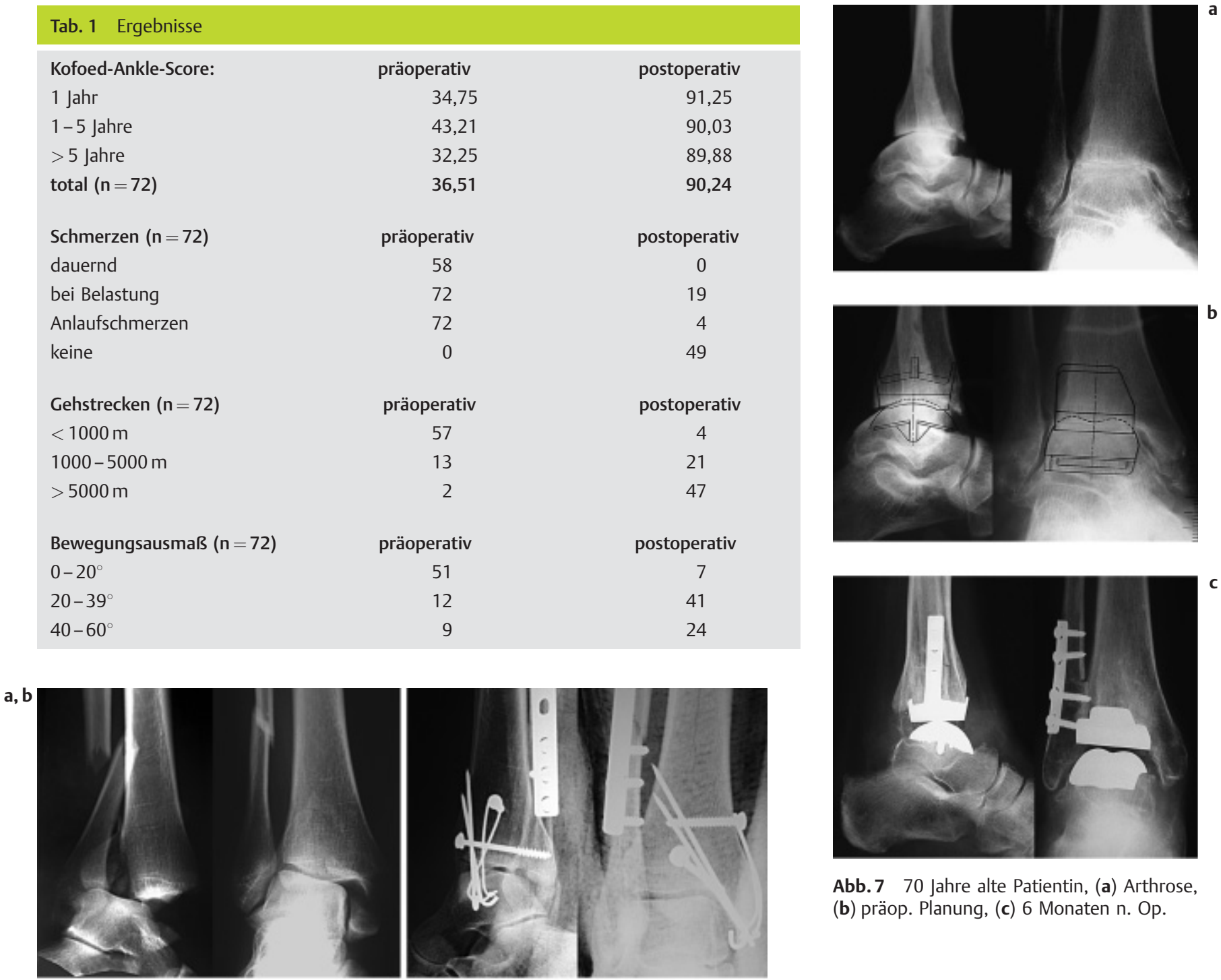

Abb. 770 Jahre alte Patientin, (a) Arthrose, (b) präop. Planung, (c) 6 Monaten n. Op.
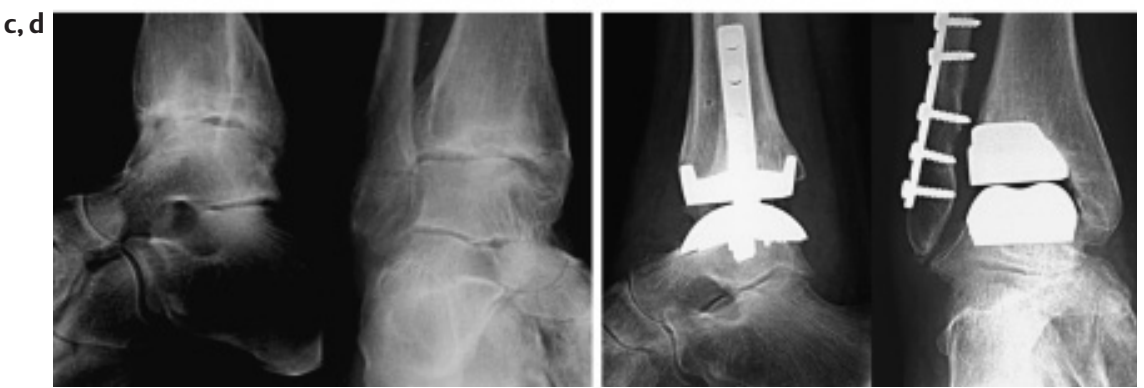

Abb. 658 Jahre alte Patientin (Hausfrau), 6 Jahre nach OSG Luxationsfraktur, (a) Unfallbilder, (b) primäre auswärtige Versorgung, (c) schmerzhafte posttraumatische Arthrose (Dauerschmerzen, Beweglichkeit $<10^{\circ}$ ), (d) 2 Jahre nach Prothesenimplantation (schmerzfreie Gehstrecke $>5 \mathrm{~km}$, Beweglichkeit $>30^{\circ}$ ).

Bei allen 3 Problemen konnte durch einen Prothesenwechsel das Verfahren beibehalten werden, ein Umstieg auf eine $\mathrm{Ar}$ throdese war in keinem Fall nötig. Das heißt von 16 implantierten Endoprothesen sind nach wie vor mit guter Funktion 13 intakt (81\%).
1-5 Jahre Implantationsdauer: In der Gruppe zwischen 1 und 5 Jahren wurden im eigenen Hause 46 Implantationen durchgeführt, die alle in unserer Nachkontrolle sind. In dieser Gruppe ist bislang kein Wechsel zu verzeichnen oder zu erwarten (100\%).
Unterschiede zwischen den einzelnen Lebensaltern waren nicht zu erkennen (die Ergebnisse waren bei Patienten unter 40 Jahren nicht anders wie in der Hauptgruppe zwischen 50 und 70 Jahren).

Komplikationen: $\mathrm{Zu}$ einer der oben genannten Umwandlungen in Arthrodesen kam es bei einer auswärts nach unfallbedingter Arthrose mit einer Endoprothese versorgten Patientin wegen schmerzhafter Verknöcherungen um den gesamten Gelenkbereich herum. Da mehrfache Abtragungen und auch eine Röntgenbestrahlung keine dauerhafte Besserung brachten, erfolgte nach 3 Jahren die Umwandlung in eine Arthrodese. Durch Einsetzen von Anteilen des Außenknöchels und Knochenmaterial aus dem Beckenkamm in das Implantatlager konnte dabei jegliche Beinverkürzung vermieden werden! 
Vor allem bei älteren Patienten gelegentlich auftretende kleinere Wundrandnekrosen heilten meist spontan ab, lediglich 5-mal wurden kleinere Lappenplastiken notwendig.

Nachoperiert innerhalb von 2 Jahren wurden 8 Patienten: 3-mal wegen eines schmerzhaften Tarsaltunnelsyndroms, 5-mal wegen schmerzhafter Verknöcherungen an der Spitze des Innenknöchels. Es handelte sich um Beschwerden, die erst durch die verbesserte postoperative Beweglichkeit und nach Beseitigung der Schmerzen im Hauptgelenkflächenbereich manifest wurden.

\section{Diskussion und Schlussfolgerung}

Fasst man die eigenen Erfahrungen seit 1990 zusammen, und berücksichtigt man die weiteren Verbesserungen der Implantate und der Implantationstechnik in den letzten 5 Jahren und die Ergebnisse allein aus dieser Zeit, so ist davon auszugehen, dass bei posttraumatischen $\mathrm{Ar}$ throsen des oberen Sprunggelenkes mehr als 75\% der Patienten schmerzfreie Gehstrecken von $5 \mathrm{~km}$ und mehr mit dem von uns verwendeten Endoprothesenmodell erlangen können. Bei den übrigen $20-25 \%$ Patienten sind es vor allem kapsuläre Probleme, die das Ergebnis beeinträchtigen (z.B. Schwellneigung oder Schmerzen nach längerer Belastung).

Weist das Implantat nach 2 Jahren eine gute Funktion auf, so kann bei heutiger Implantationstechnik und Implantatqualität nach den bisherigen Erfahrungen und den über 10-jährigen Verlaufsbeobachtungen mit einer weiteren Überlebensrate von 20 Jahren gerechnet werden.

Dies gilt auch für Patienten mit einem Lebensalter von 20 bis 50 Jahren und aktiver Berufstätigkeit (Abb.5)!
Die Vorteile gegenüber einer Arthrodese sind bei dieser Gruppe besonders auffallend: Vermeidung von Sekundärarthrosen in Nachbargelenken, bessere Beweglichkeit und Gehfähigkeit, meist schnellere Wiedereingliederung in den Arbeitsprozess (eigene Patienten nach durchschnittlich 3 Monaten) [5,8].

Vergleicht man die eigenen Ergebnisse mit den Angaben in Publikationen über andere Endoprothesenmodelle (vor allem über die STAR-Endoprothese liegenden aussagekräftige Publikationen vor), liegen die Ergebnisse etwa im gleichen Bereich $[6,10]$.

Dabei ist zu berücksichtigen, dass allerdings bei Meniskusendoprothesen das Krankengut stärker selektioniert ist, da für diese Endoprothesen eine intakte Sprunggelenksgabel vorausgesetzt werden muss und größere Achsenfehler als Kontraindikation gelten und dass für einen Teil dieser Prothesen noch keine 5- oder 10-Jahres-Ergebnisse vorliegen.

Die Implantation von Sprunggelenksendoprothesen ist wegen zahlreicher Variationen bezüglich Knochenstruktur, arthrotischer Deformierung und anatomischer Form eine anspruchsvolle Operationstechnik, die eine spezielle Erfahrung und eine gute präoperative (Abb. 7) Planung voraussetzt.

\section{Literatur}

${ }^{1}$ Botton-Maggs BG, Sudlow RA, Freeman MAR. Total ankle arthroplasty A long-term review of the London Hospital experience. J bone Jt surg 1985; 67-B: 785-790

${ }^{2}$ Büchel EF, Pappas MJ, Iorio MI. New Jersey low contact stress total ankle replacement: Biomechanical ratonale and Review of 23 cementless cases. Foot Ankle 1988; 8: $279-$ 289

${ }^{3}$ Carlsson AS, Hericson A, Linder L, et al. A survival analysis of 52 Bath \& Wessex ankle replacements (A clinical and radiographic study in patients with rheumatoid arthritis and a critical review of the literature). The Foot 1994; 4: 34-40

${ }^{4}$ Kitaoka HB, Patzer GL, Ilstrup DM, Wallrichs SL. Survorschip analysis of the Majo total ankle arthroplasty. J bone Jt Surg 1994; 76-A: 974-979

${ }^{5}$ Kofoed H, Stürup J. Comparison of ankle arthroplasty and arthrodesis. A prospective series with long therm follow-ups. Foot 1994; 4: 6-9

${ }^{6}$ Kofoed H. Die Entwicklung der Sprunggelenksarthroplastik. Orthopäde 1999; 28 : 804-811.

${ }^{7}$ Lord G, Marotte JH. Prothese totale de cheville Technique et premiers resultats apropos de 12 observations. Rev chir orthop 1973; 59: 139-151

${ }^{8}$ McGuire MR, Kyle RF, Gustilo RB, Premer RF. Comparative Analysis of ankle arthroplasty versus ankle arthrodesis. Line Orthop 1988; 226: $174-181$

${ }^{9}$ Rudigier J, Grundei H, Menzinger F. Prosthetic Replacement of the Ankle in Posttraumatic Arthrosis. Eur J Trauma 2001; 27: 66 74

${ }^{10}$ Thermann H, Saltzmann CL. Endoprothetischer Ersatz des oberen Sprunggelenkes. Unfallchirurg 2002; 1005: 496-510

Prof. Dr. med. Jürgen Rudigier Chefarzt

Dr. med. Felix Menzinger

Oberarzt

Klinik für Unfall und Handchirurgie Klinikum Offenburg

Ebertplatz 12

D-77654 Offenburg 